\title{
REVERSIBLE VISCERAL ISCHEMIA DETECTED BY TRANSESOPHAGEAL ECHOCARDIOGRAPHY AND NEAR-INFRARED SPECTROSCOPY
}

Kazumasa Orihashi, MD, Yuichiro Matsuura, MD, Taijiro Sueda, MD, Masanobu Watari, MD, and Kenji Okada, MD,

Hiroshima, Japan

From the First Department of Surgery, Hiroshima University School of Medicine, Hiroshima, Japan.

Received for publication Aug 26, 1999; accepted for publication Oct $7,1999$.

Address for reprints: Kazumasa Orihashi, MD, First Department of Surgery, Hiroshima University School of Medicine, Kasumi 1-2-3, Minami-ku, Hiroshima, 734-8551 Japan (E-mail: kaori@mcai.med.hiroshima-u.ac.jp).

J Thorac Cardiovasc Surg 2000;119:384-6

Copyright $@ 2000$ by Mosby, Inc.

$0022-5223 / 2000 \$ 12.00+0 \quad \mathbf{1 2 / 5 4 / 1 0 3 6 0 1}$
Visceral ischemia in aortic dissection leads to a poor prognosis if diagnosis and treatment are delayed. However, neither angiography nor computed tomography (CT) is feasible when the patient needs immediate surgery and is in unstable hemodynamic condition.

Recently we reported a technique of visualizing visceral arteries with the use of transesophageal echocardiography (TEE).$^{1,2}$ Meanwhile, near-infrared spectroscopy (NIRS) has been introduced for noninvasively monitoring regional oxygen saturation $\left(\mathrm{rSO}_{2}\right)$ in the brain. . $^{3-5}$

We report a case of transient visceral ischemia caused by aortic dissection in which use of TEE and NIRS for diagnosis was suggested.
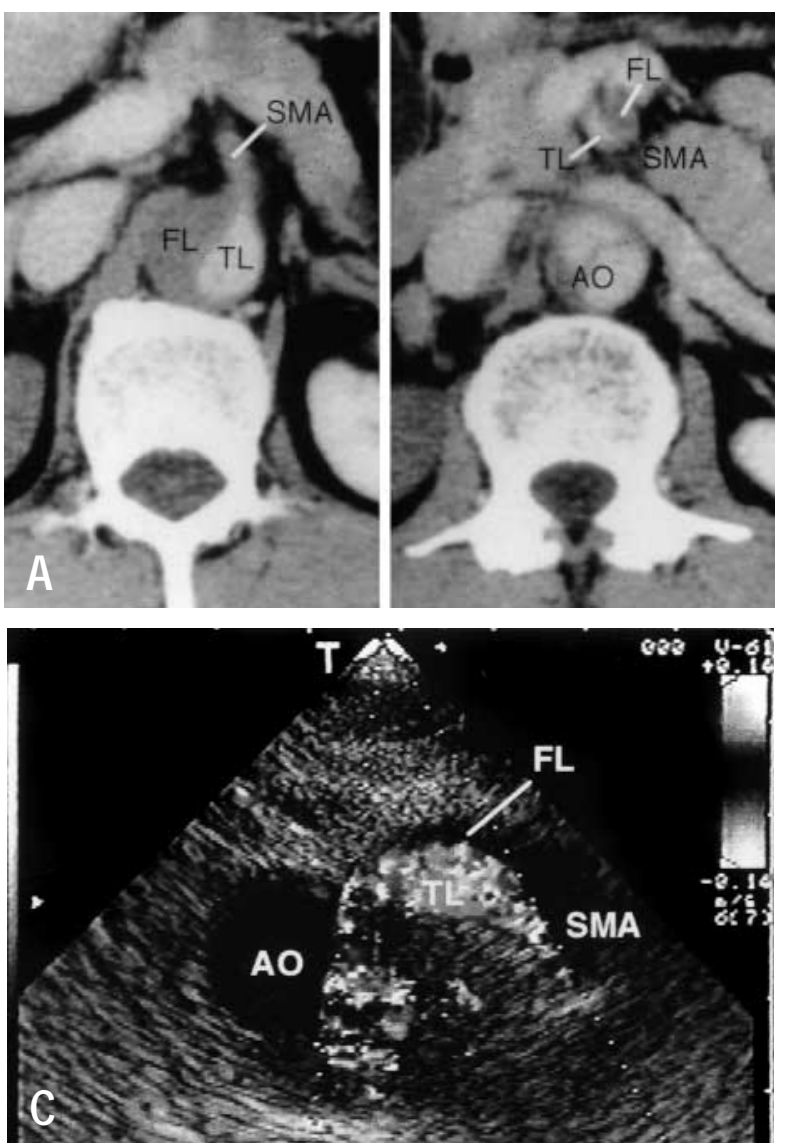

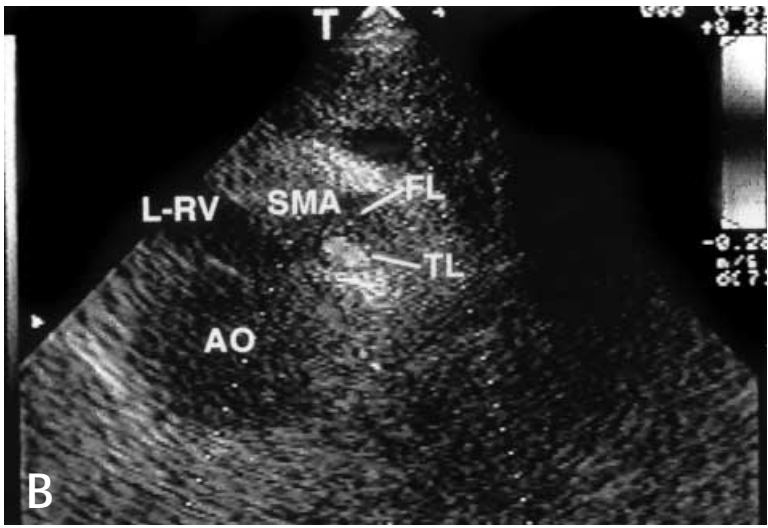

Fig 1. Changes of CT and TEE findings. CTs on the first day (A) and fourth day (D); TEE on the second day (B) and third day $(\mathbf{C})$. The true lumen $(T L)$ of the superior mesenteric artery (SMA) was narrowed by thrombosed false lumen $(F L)$ in $(\mathbf{A})$ and $(\mathbf{B})$, then improved $(\mathbf{C}$ and $\mathbf{D})$. $A O$, Aorta; $L-R V$, left renal vein. 
Table I. Changes of $r \mathrm{SO}_{2}$ with laboratory data

\begin{tabular}{|c|c|c|c|c|c|}
\hline & \multicolumn{5}{|c|}{ Time from onset } \\
\hline & $3 h$ & $16 \mathrm{~h}$ & 1 day & $2 d$ & $7 d$ \\
\hline Epigastrium (left lobe) & 75 & 75 & 69 & 67 & 73 \\
\hline Liver (right intercostal) & 70 & 70 & 69 & 70 & 72 \\
\hline Above navel & 73 & 75 & 66 & 70 & 71 \\
\hline Below navel & & 64 & 67 & 70 & 69 \\
\hline Right lower quadrant & 64 & 61 & 64 & 69 & 71 \\
\hline Left lower quadrant & 62 & 64 & 61 & 70 & 68 \\
\hline Right thigh & 62 & 66 & 64 & 62 & 78 \\
\hline \multirow[t]{3}{*}{ Left thigh } & 76 & 75 & 66 & 61 & 71 \\
\hline & \multicolumn{5}{|c|}{ Arterial oxygen saturation (\%) } \\
\hline & 98 & 98 & 91 & 95 & 96 \\
\hline $\mathrm{pH}$ & 7.302 & 7.264 & 7.342 & 7.392 & 7.426 \\
\hline Base excess $(\mathrm{mEq} / \mathrm{L})$ & -1.5 & -5.2 & 1.2 & 0.2 & 1.1 \\
\hline LDH & 474 & & 7335 & 6210 & 1818 \\
\hline $\mathrm{CK}$ & 67 & & 409 & 676 & 366 \\
\hline Lactate & 10.6 & 21.7 & 7.69 & 3.81 & 4.94 \\
\hline Dorsalis pedis artery & $\mathrm{R}<\mathrm{L}$ & $\mathrm{R}=\mathrm{L}$ & $\mathrm{R}=\mathrm{L}$ & $\mathrm{R}=\mathrm{L}$ & $\mathrm{R}=\mathrm{L}$ \\
\hline Coldness of leg & $\mathrm{R}>\mathrm{L}$ & $\mathrm{R}>\mathrm{L}$ & - & - & - \\
\hline
\end{tabular}

$L D H$, Lactic dehydrogenase; $C K$, creatine kinase; $R$, right; $L$, left. The $\mathrm{rSO}_{2}$ was lower by more than $10 \%$ at lower abdomen and right thigh (boldface numbers) than at the epigastrium. One day later the difference became less than $10 \%$. The $\mathrm{rSO}_{2}$ is influenced by arterial oxygen saturation.

Clinical summary. A 61-year-old man was admitted after an acute onset of back pain with a blood pressure $221 / 67 \mathrm{~mm}$ $\mathrm{Hg}$. Pulsation was noted at the left femoral artery but not at the right. He also had pain of the right lower extremity. CT revealed DeBakey type IIIb aortic dissection with thrombosed false lumen or intramural hematoma, which led to occlusion of the right renal and right common iliac arteries. The celiac and left renal arteries were intact. Incomplete filling was noted at the proximal portion of superior mesenteric artery (Fig 1, A).

A 40-mm sensor of TOS-96 (TOSTEC Co Ltd, Tokyo, Japan), an NIRS system that provides $\mathrm{rSO}_{2}$ at 2 to $3 \mathrm{~cm}$ depth, was placed on multiple sites of the abdominal wall and thighs. The $\mathrm{rSO}_{2}$ was recorded when the value became stable after 1 minute (Table I). It was lower at the right and left lower quadrants and right leg than at the epigastric region by more than $10 \%$. Sixteen hours from onset, the $\mathrm{rSO}_{2}$ remained low. The patient still had abdominal pain and also had metabolic acidosis ( $\mathrm{pH} 7.26$ ) with a base excess of $-5.2 \mathrm{mEq} / \mathrm{L}$.

TEE showed an aortic dissection that extended into the superior mesenteric artery, narrowing its lumen to smaller than $50 \%$ because of the thrombosed false lumen (Fig 1, B). During medical therapy, the $\mathrm{rSO}_{2}$ gradually improved.

On the second day, TEE revealed that the true lumen had become larger (Fig 1,C). The $\mathrm{rSO}_{2}$ became equal at every site, associated with improved laboratory data. CT on the fourth day showed that the superior mesenteric and right iliac arteries had no filling defects (Fig 1,D). The patient had no pain. His course thereafter was uneventful.

Discussion. Both TEE findings and NIRS data were total- ly compatible with symptoms, laboratory data, and CT findings. This case suggests possible application of TEE and NIRS for diagnosing and monitoring visceral ischemia.

TEE detects dissection into the visceral arteries, as well as impaired perfusion. The superior mesenteric artery can be visualized with TEE by means of a specific technique in more than $95 \%$ of patients having cardiovascular surgery. ${ }^{1,2}$ Visualization is not disturbed by gas in the intestines and is applicable repeatedly at the bed side, providing real-time information. Drawbacks include possible invasiveness for conscious patients and nonvisualization of iliac arteries.

The merits of NIRS are that $\mathrm{rSO}_{2}$ in the body can be obtained continuously and noninvasively with minimal influence of oxygenation in the tissue adjacent to the sensor. However, no data are available regarding the normal range and critical value of $\mathrm{rSO}_{2}$ for visceral ischemia. Because the $\mathrm{rSO}_{2}$ can vary among individuals and be affected by hemoglobin level, arterial oxygenation, and other factors, we assessed the relative decrease of $\mathrm{rSO}_{2}$ compared with $\mathrm{rSO}_{2}$ at the epigastric region. Ultrasonography showed that the left lobe of the liver was at the sampling region. The $\mathrm{rSO}_{2}$ was assumed to be normal at the liver since the celiac artery was intact. The $\mathrm{rSO}_{2}$ at the right and left lower quadrants should reflect oxygenation in the intestinal wall unless bleeding occurs in the peritoneal cavity or intestinal tract. However, a false negative result is probable when ischemia is not diffuse but regional.

This case suggested that TEE and NIRS enable noninvasive diagnosis of visceral ischemia. However, further investigation is mandatory for evaluating its accuracy in a larger series and in regional necrosis of the intestine. 


\section{REFERENCES}

1. Orihashi K, Matsuura Y, Sueda T, Shikata H, Morita S, Hirai S, et al. Abdominal aorta and visceral arteries visualized with transesophageal echocardiography during operations on the aorta. $\mathbf{J}$ Thorac Cardiovasc Surg 1998;115:945-7.

2. Orihashi K, Matsuura Y, Sueda T, Shikata H, Morita S, Hirai S, et al. Abdominal aorta and visceral arteries visualized by transgastric echocardiography: technical considerations. Hiroshima J Med Sci 1997;46:151-7.

3. Kurth CD, Steven JM, Nicolson SC. Cerebral oxygenation during pediatric cardiac surgery using deep hypothermic circulatory arrest. Anesthesiology 1995;82:74-82.

4. Kirkpatrick PJ, Lam J, Al-Rawi P, Smielewski P, Czosnyka MIT. Defining thresholds for critical ischemia by using nearinfrared spectroscopy in the adult brain. J Neurosurg 1998;89: 389-94.

5. Katoh T, Esato K, Gohra H, Hamano K, Fujimura Y, Zempo N, et al. Evaluation of brain oxygenation during selective cerebral perfusion by near-infrared spectroscopy. Ann Thorac Surg 1997;64: 432-6. 